\title{
3. Zum Europagedanken im innerdeutschen Widerstand: ein Vergleich
}

Als die ersten Nachrichten über das mißlungene Attentat vom 20. Juli 1944 den Atlantik überquert hatten, urteilte der Sozialdemokrat Rudolf Katz: „Es handelt sich nicht um eine aktuelle Revolte, nicht um einen Putsch-Versuch einer größeren Machtgruppe. Es handelt sich vielmehr um eine ,Purge“" ${ }^{\text {“1056, }}$ eine gezielt herbeigeführte Reinigungsaktion des Hitler-Regimes. Sicherlich unterschätzte Katz die weitverzweigten Verflechtungen der mit dem 20. Juli 1944 mehr oder weniger in Verbindung stehenden Widerstandsgruppen innerhalb Deutschlands. Eines aber lassen Katz' Worte deutlich erkennen: das tief verwurzelte Mißtrauen der Emigranten gegenüber dem innerdeutschen Widerstand, soweit er nicht unmittelbar in den Bevölkerungsgruppen wurzelte, die mit dem Exil in organisatorischer Verbindung standen. Die Widerstandsgruppen, die mit dem Attentat des Obersten von Stauffenberg in Verbindung standen, rekrutierten sich jedoch vorwiegend aus den alten Eliten eines Teils der Militärführung, des diplomatischen Korps, der Verwaltung und der Kirchen. Deren „Verbindung zu der großen Welt ${ }^{\star 1057}$ erschöpfte sich in unregelmäßigen Bemühungen, mit Vertretern der alliierten Regierungen ins Gespräch zu kommen und ihnen Denkschriften zur künftigen Rolle Deutschlands und Europas zu übermitteln. Über organisatorische Verbindungen zum Ausland oder gar zum deutschen Exil verfügten diese Gruppen nicht. Selbst die an den Kreisen um Moltke und Goerdeler beteiligten Sozialdemokraten wie Carlo Mierendorff, Adolf Reichwein, Theodor Haubach, Julius Leber oder Wilhelm Leuschner standen in eher losem Kontakt zum Exilvorstand der Sopade. Der spärliche Kontakt zwischen den genannten Sozialdemokraten und der Exilvertretung ihrer Partei ist nicht zuletzt auf die bis 1938 erfolgte gründliche Zerschlagung der grenzüberschreitenden illegalen Kommunikationsstrukturen durch die Gestapo sowie zu einem nicht geringen Anteil auf die Eigenwilligkeit dieser sozialdemokratischen Individualisten zurückzuführen. Ohnehin hatte es selbst zwischen den sozialdemokratischen und sozialistischen Exilvertretungen und ihren innerdeutschen Verbindungsgruppen zu keinem Zeitpunkt ein tatsächliches „Anleitungsverhältnis" 1058 gegeben.

Mithin kann festgehalten werden, daß sich die zwei von Beginn an ungleichen Seiten der deutschen Widerstandsmedaille, der innerdeutsche Widerstand einerseits und das deutsche Exil andererseits, im Kriegsverlauf weiter entfremdeten, so daß im Jahre 1944 schließlich für die jeweils andere Seite

1056 Katz, Der zwanzigste Juli. Putschversuch oder Purge?, in: NVZ, 13. Jg., Nr. 31 (29. 7. 1944), S. 4.

1057 So der Titel einer Abhandlung von KLEMPERER, „Verbindung zu der großen Welt ${ }^{4}$ 1990.

1058 MeHringer, Anmerkungen zum Verhältnis von Exil und innerdeutschem Widerstand 1998, S. 106. 
nur noch Mißtrauen aufgebracht wurde. Während das Exil, soweit es überhaupt über Aktivitäten des innerdeutschen Widerstandes unterrichtet war, die Verflechtungen der überwiegend bürgerlichen Widerstandskämpfer mit dem nationalsozialistischen System kritisch bis ablehnend beurteilte, war für die große Mehrheit der inneren Widerstandsgruppen schon der Entschluß zur Emigration unverständlich: „Besser, Talleyrandsche Methoden' anwenden, seine wahre Gesinnung also tarnen, sich demütigen lassen und ein Doppelspiel treiben, aber heimlich Widerstand leisten, statt zu emigrieren - so lautete selten einmütig die Auffassung der Verschwörer des 20. Juli. “1059 Insofern verwundert es nicht, daß sich Exil und innerer Widerstand als zwei voneinander völlig unabhängige Handlungsebenen erwiesen, die sich durch ,jeweils eigenständige Interessen, Kampfformen, Bewußtseinslagen und politisch-intellektuelle Leistungen " 1060 auszeichneten.

Im folgenden kann es nicht darum gehen, einen ausführlichen Vergleich des Europadiskurses im Exil mit dem Europadiskurs im innerdeutschen oder gar europäischen Widerstand anzustellen, denn dieser umfassenden Fragestellung kann nur monographisch angemessen begegnet werden. Vielmehr soll ein kursorischer Überblick über zentrale Forderungen in den Europadebatten des innerdeutschen Widerstands die Unterschiede und Gemeinsamkeiten mit dem deutschen Exil hervorheben. So wird der Europadiskurs des Exils in den historischen Zusammenhang der gesamten deutschen Widerstandsbewegung eingeordnet und zugleich die trotz vielfältiger Irrtümer und Mißverständnisse nicht zu leugnende Leistung des Exils als bedeutendem Teil des aktiven Widerstandes offenbart.

Ungeachtet der recht großen Zahl an Widerstandsgruppen innerhalb des nationalsozialistischen Deutschlands reduziert sich die Auseinandersetzung mit programmatischen Fragestellungen der Außenpolitik auf nur wenige Gruppen des bürgerlichen Widerstandes. Die sozialistischen und sozialdemokratischen Widerstandsgruppen konzentrierten sich auf die äußert schwierige Organisation des täglichen illegalen Widerstandes vor Ort und in den Betrieben und überließen den programmatischen Teil der Widerstandsarbeit ihren Leitungsgremien im Ausland. Der anfangs starke und weit verzweigte kommunistische Widerstand innerhalb Deutschlands zeigte wenig Interesse an außenpolitischen Überlegungen und richtete sich - trotz divergierender Ansichten einzelner - nach den Vorgaben aus Moskau. Auch der heterogene Kreis um Arvid Harnack und Harro Schulze-Boysen, der von der Gestapo dem europäischen Widerstandsnetz der Roten Kapelle zugerechnet worden war, widmete sich nicht explizit der Europaproblematik.

Unter den innerdeutschen Widerstandsgruppen, die sich ausführliche Erörterungen der außenpolitischen Rahmenbedingungen und Zukunftssze-

1059 EHLERS, Technik und Moral 1964, S. 28.

1060 MeHRINGER, Anmerkungen zum Verhältnis von Exil und innerdeutschem Widerstand 1998, S. 106. 
narien leisteten, ragen zwei Repräsentanten des zivilen bürgerlichen Widerstandes besonders hervor: die sogenannte Honoratiorengruppe um den ehemaligen Leipziger Oberbürgermeister Carl Goerdeler und der Kreisauer Kreis um Helmuth von Moltke und Peter Yorck von Wartenburg.

Die Honoratiorengruppe, deren zentrale Figur, der Nationalliberale Carl Goerdeler, als „Magnet und Motor" der Opposition bezeichnet worden ist $^{1061}$, setzte sich vorwiegend aus ehemaligen und im Amt verbliebenen Verantwortungsträgern aus Verwaltung und Politik zusammen. Zu den prominenten Mitgliedern zählten der ehemalige Botschafter Ulrich von Hassell sowie die beiden ursprünglich begeisterten Nationalsozialisten Johannes Popitz, preußischer Finanzminister, und der Staatsrechtler Jens Jessen. Die Arbeiterbewegung war durch den christlichen Gewerkschafter Jakob Kaiser und den sozialdemokratischen Gewerkschafter Wilhelm Leuschner vertreten. Auch der ehemalige Chef des Generalstabs des Heeres, Ludwig Beck, kann zum Goerdeler-Kreis gezählt werden. Goerdeler selbst war 1940 aus Protest gegen die Entfernung des Leipziger Mendelssohn-Denkmals zurückgetreten. Nicht nur das antisemitische Motiv, sondern vor allem das eigenmächtige Handeln der örtlichen NSDAP-Führer während seiner dienstlich bedingten Abwesenheit hatten Goerdeler gegen die Partei in Stellung gebracht, zumal ihm als überzeugtem Kommunalpolitiker die Eigenständigkeit des Bürgermeisters gegenüber der Partei viel bedeutete ${ }^{1062}$.

Der Kreisauer Kreis rekrutierte sich ebenfalls aus den Reihen der alten Eliten, repräsentierte jedoch deren jüngere Generation ${ }^{1063}$. Neben dem „Herzen "1064 des Kreises, Peter Yorck von Wartenburg, und dem als "Beweger“ 1065 titulierten Helmuth von Moltke, den George F. Kennan als „eine[n] der wenigen protestantischen Märtyrer unserer Tage“ bezeichnete ${ }^{1066}$, gehörten zu dieser bedeutenden Widerstandsgruppe die Diplomaten Adam v. Trott zu Solz und Hans-Bernd v. Haeften, die höheren Verwaltungsbeamten Horst von Einsiedel und Carl Dietrich von Trotha, der sozialdemokratische Reformpädagoge Adolf Reichwein, die Hofgeismarer Carlo Mierendorff und Theodor Haubach, Kardinal Faulhaber, der Jesuitenpater Alfred Delp und die protestantischen Theologen Harald Poelchau und Eugen Gerstenmaier.

Während der Goerdeler-Kreis seine geistigen Wurzeln recht homogen im nationalkonservativen Denken des 19. Jahrhunderts fand und sich in bestimmten Punkten mit den Ansichten des Staatsrechtlers Carl Schmitt einig wußte, liefen im Kreisauer Kreis „vielfältige geistesgeschichtliche und bio-

1061 Vgl. FEST, Staatsstreich 1994, S. 148.

1062 Vgl. RoON, Widerstand 1979, S. 140.

1063 Vgl. MEHRINGER, Widerstand und Emigration 1997, S. 193.

1064 Vgl. FEST, Staatsstreich 1994, S. 158.

1065 Ibid.

1066 KenNaN, Memoiren 1971, S. $128 \mathrm{ff}$. 
graphische Entwicklungslinien ineinander“1067. In seiner Studie über „Widerstand und Emigration" nennt Mehringer vier bestimmende Entwicklungslinien ${ }^{1068}$ : die Reformpädagogik mit den Bildungsidealen der Jugendbewegung, den religiösen Sozialismus nach Paul Tillich ${ }^{1069}$, die katholisch geprägten sozialreformerischen Traditionen und die sozialistische Tradition.

Lange Zeit arbeiteten der Kreisauer Kreis und der Goerdeler-Kreis trotz individueller Kontakte getrennt voneinander, bis sie sich am 8. Januar 1943 $\mathrm{zu}$ einem ersten Gedankenaustausch trafen. Wenngleich die Vertreter des Kreisauer Kreises jedenfalls in einer Personalie einlenkten und Goerdeler für den Posten des künftigen Reichskanzlers akzeptierten, traten bei dem Treffen viele Bruchlinien offen zutage, die man auch in der nachfolgenden Zeit nicht auszuräumen vermochte.

Während Goerdeler mit dem militärischen Widerstand in enger Verbindung stand, waren die Kontakte zwischen dem Militär und dem Kreisauer Kreis, der zumindest in Teilen ein fundamental anderes, strikt gewaltloses Widerstandsverständnis hatte ${ }^{1070}$, weniger ausgeprägt. Erst die prozessuale Zusammenlegung in den Volksgerichtshofsprozessen nach dem Attentatsversuch vom 20. Juli 1944 stellte den Goerdeler-Kreis, den Kreisauer Kreis und den militärischen Widerstand unzutreffend als eine einheitliche Widerstandsbewegung dar. Doch entsprach dies auch der Wahrnehmung Hitlers, der nach Einsichtnahme in die aufgefundenen Tagebücher von Admiral Canaris seine ursprüngliche, in der Radiorede vom 21. Juli 1944 veröffentlichte These von einigen wenigen Verschwörern widerlegt zu sehen meinte. Daraufhin gab er in einem Zustand gesteigerten Verfolgungswahns den Befehl zur Aburteilung der inhaftierten Verdächtigen, die sogleich unter gröbster Mißachtung selbst der NS-Strafgesetze von Standgerichten zum in der Regel sofort vollstreckten Tod durch den Strang verurteilt wurden. Helmuth v. Moltke, den der amerikanische Diplomat George F. Kennan als "tiefreligiöse[n] Mann von ungewöhnlichem moralischem Mut" charakterisierte, bedauerte zutiefst die unterschiedlose Vermischung des Kreisauer Kreises und seiner eigenen Person mit dem "Goerdeler-Mist“ und dem gewaltsamen Vorgehen Stauffenbergs ${ }^{1071}$.

In ihrer europapolitischen Programmatik standen sich die Kreisauer und die "Honoratioren“ nahezu diametral gegenüber. Moltke, Trott und andere forderten einen souveränen europäischen Bundesstaat, während die Honoratiorengruppe offen für ein Europa unter deutscher Hegemonie warb. Erst als Goerdeler begann, den deutschen Führungsanspruch sukzessive durch die

1067 Vgl. MEHRINGER, Widerstand und Emigration 1997, S. 193.

1068 Ibid.S. $193 \mathrm{f}$.

1069 Die Ideen des religiösen Sozialismus wurden primär durch den protestantischen Gefängnispfarrer Harald Poelchau in den Kreis eingebracht; vgl. ibid. S. 194.

$1070 \mathrm{Zu}$ diesem tragenden Unterschied zwischen der Goerdeler-Gruppe und dem Kreisauer Kreis vgl. FEST, Staatsstreich 1994, S. 163.

1071 Zit. n. FEST, Staatstreich 1994, S. 315. 
Vision eines europäischen Friedensbundes ohne Hegemonialmacht zu ersetzen, kamen sich Moltke und Goerdeler in der Europafrage ein wenig näher.

Für die europäischen Neuordnungspläne des Kreisauer Kreises sind vor allem die Denkschriften und Notizen von Moltke und Trott zu Solz sowie die Ergebnisse der drei Haupttagungen vom Mai 1942, Oktober 1942 und Juni 1943 ausschlaggebend. Konzipiert als Alternative zum totalen Machtanspruch des „Dritten Reiches“1072, hatten die Kreisauer Europadebatten keineswegs zum Ziel, ein konsensorientiertes Minimalprogramm zu entwickeln. Vielmehr bemühte man sich um einen christlich-sozialistischen Idealentwurf ${ }^{1073}$, der sich im Vergleich zu den Überlegungen der Honoratiorengruppe insgesamt als differenzierter und sensibler erwies ${ }^{1074}$.

Bestimmendes Motiv der Kreisauer Europavorstellungen war „die verpflichtende Besinnung des Menschen auf die göttliche Ordnung, die sein inneres und äußeres Dasein trägt"1075. Daraus folgte die zentrale politische Forderung, die den Kreisauer Kreis von den meisten innerdeutschen Widerstandsgruppen unterscheidet ${ }^{1076}$ : die Überwindung des übersteigerten $\mathrm{Na}$ tionalismus zugunsten einer einheitlichen Souveränität für Europa ${ }^{1077}$. Moltke, der ohne Wehmut und Bedauern Deutschlands ,totale[m] Zusammenbruch als nationale Einheit ${ }^{* 1078}$ gelassen entgegensah, erhoffte sich von einem vereinigten Europa das Ende des Nationalismus, der Gewaltpolitik und des Rassegedankens sowie der Gewalt des Staates über den einzelnen ${ }^{1079}$. In Übereinstimmung mit den Europakonzepten des deutschen Exils begrüßten die Kreisauer die Möglichkeit, innerhalb eines europäischen Bundesstaates die zahlreichen Minderheitenfragen friedlich zu lösen und die vielfältigen Kriegsherde endgültig zu beseitigen. Das Programm war darauf ausgelegt, strukturelle Bedingungen für einen nachhaltigen Frieden in Europa zu schaffen und zugleich die gemeinsame Aufbauarbeit nach dem Krieg als Chance für eine weitergehende Kooperation zu begreifen ${ }^{1080}$. Dies setzte jedenfalls nach der Überzeugung des inneren Kreises eine sichtbare Einschränkung der nationalstaatlichen Souveränität durch eine wirksame internatio-

1072 MOMMSEN, Künftige Neuordnung 1994, S. 246; siehe auch Ders., Alternative zu Hitler 2000, S. 207-229.

1073 FEST, Staatsstreich 1994, S. 160 sieht darin sogar einen „stark utopische[n] Zug“. 1074 Vgl. MeHringer, Widerstand und Emigration 1997, S. 193.

1075 Grundsätze für die Neuordnung, 9. 8. 1943, abgedruckt in: LIPGENS, Föderationspläne 1968, S. 153 ff. (153).

1076 Vgl. RooN, Neuordnung im Widerstand 1967, S. 452.

1077 Moltke, Ausgangslage, Ziele und Aufgaben, 24. 4. 1941, abgedruckt in: RooN, Neuordnung im Widerstand 1967, S. 507-517 (512). Das Dokument findet sich auch in der Quellensammlung von LIPGENS, Föderationspläne 1968, S. 111-117.

1078 Moltke an Lionel Curtis, [Mitte Mai?]1942, abgedruckt in: LIPGENS, Föderationspläne 1968, S. 128-130 (129).

1079 Moltke, Ausgangslage, Ziele und Aufgaben, 24. 4. 1941, abgedruckt in: RooN, Neuordnung im Widerstand 1967, S. 510.

1080 ROON, Neuordnung im Widerstand 1967, S. 454. 
nale Instanz voraus, die soziale Gerechtigkeit und „Ordnung bei einem Maximum von Freiheit" gewährleisten müsse ${ }^{1081}$.

Wie viele Emigranten hatten insbesondere Moltke und die Kreisauer Sozialdemokraten die Dringlichkeit einer Europäisierung des Bewußtseins erkannt. Sie forderten die Schaffung einer gemeinsamen christlich-humanistisch-sozialistischen Grundauffassung ${ }^{1082}$, denn: „Es hat nun gar keinen Sinn, die einheitliche Souveränität herzustellen, wenn es nicht gelingt, dem europäischen Staatsvolk die einheitliche Grundauffassung wiederzugeben, die Jahrhunderte lang verschüttet gewesen ist. " 1083 Moltke dachte dabei an eine Rückbesinnung auf die gemeinsame gesamteuropäische Kultur und Tradition. In diesem Zusammenhang stehen seine berühmten Worte aus einem Brief an Lionel Curtis: „Für uns ist Europa nach dem Kriege weniger eine Frage von Grenzen und Soldaten, von komplizierten Organisationen oder großen Plänen. Europa nach dem Kriege ist die Frage: Wie kann das Bild des Menschen in den Herzen unserer Mitbürger aufgerichtet werden.“1084

Charakteristisches Kernelement des „europäischen Internationalismus" 1085 der Kreisauer war zweifellos die weitreichende Souveränität, die man dem europäischen Bundesstaat zugestehen wollte. In diesem Punkt wurden selbst die Konzepte einiger überzeugter Europaföderalisten des Exils übertroffen. Gleichwohl darf nicht übersehen werden, daß der Grad der Einschränkung nationalstaatlicher Souveränität innerhalb des Kreisauer Kreises umstritten war. Am weitesten ging Moltke, dessen Konzept der kleinen Gemeinschaften zur Wiederherstellung und Stärkung des individuellen Verantwortungsgefühls ${ }^{1086} \mathrm{im}$ Exil nur bei Friedrich Darmstaedter wiederzufinden ist $^{1087}$. Moltke forderte wie Darmstaedter die Zerschlagung der Nationalstaaten und die Neueinteilung des europäischen Kontinents - ohne Großbritannien und die Sowjetunion ${ }^{1088}$ - in historisch gewachsene, nicht souveräne

1081 Zit. n. RooN, Neuordnung im Widerstand 1967, S. 469. Nach Mommsens Einschätzung verstanden sich deshalb zumindest die Angehörigen des inneren Kreises als Revolutionäre; vgl. MOMMSEN, Künftige Neuordnung 1994, S. 260.

1082 Vgl. RoON, Neuordnung im Widerstand 1967, S. 456.

1083 Moltke, zit. n. RoON, Neuordnung im Widerstand 1967, S. 455.

1084 Moltke an Curtis, [Mitte Mai?] 1942, abgedruckt in: LIPGENS, Föderationspläne 1968, S. 130.

1085 GramL, Außenpolitische Vorstellungen 1984, S. 117.

1086 Moltke, Ausgangslage, Ziele und Aufgaben, 24.4. 1941, abgedruckt in: ROON, Neuordnung im Widerstand 1967, S. 510.

1087 Vgl. dazu das Kapitel IV.2.5.

1088 Die Beteiligung Großbritanniens und der Sowjetunion wurde jedoch lebhaft diskutiert. So sprach sich Moltke wegen der britischen Verpflichtungen gegenüber dem Empire gegen eine Einbindung Großbritanniens aus, während Trott dafür war. Eine Einbindung der Sowjetunion dagegen wurde allgemein abgelehnt, auch wenn die Notwendigkeit freundschaftlicher Zusammenarbeit angesichts der zu erwartenden starken Position Rußlands auf dem europäischen Kontinent unterstrichen wurde, vgl. RoON, Neuordnung im Widerstand 1967, S. 460 f. Es sei allerdings hingewiesen auf Moltkes vor dem 22. 6. 1941 geäußerte Idee einer Integration des europäischen Teils der Sowjet- 
Selbstverwaltungseinheiten etwa vergleichbarer Größe ${ }^{1089}$. Damit könnten die nationalen Egoismen endgültig überwunden, das deutsch-französische Übergewicht gebrochen und die Entstehung neuer Hegemonien verhindert werden 1090 . In der Konsequenz bedeutete dies die völlige Aufhebung der nationalstaatlichen Souveränität und die Schaffung einer einheitlichen europäischen Souveränität „von Portugal bis zu einem möglichst weit nach Osten vorgeschobenen Punkt, bei Aufteilung des ganzen Festlandes in kleinere nicht-souveräne Staatsgebilde, die unter sich Verflechtungen politischer Art haben“ ${ }^{\text {1091. }}$.

Moltkes weitgehende Neuordnungsvorstellungen stießen bei den meisten Kreisauern allerdings auf Ablehnung, wenngleich man sich einig war, daß die dominierende Stellung der europäischen Großmächte gebrochen und absolute Souveränität der Nationalstaaten relativiert werden müsse. Stellvertretend für die Mehrheit der Kreisauer umschrieb der Mitarbeiter des Auswärtigen Amtes, Adam von Trott zu Solz, die gestufte Einschränkung der nationalstaatlichen Souveränität mit der Forderung nach „Zusammenlegungen der administrativen Souveränität auf Teilgebieten des Lebens“1092. Dem lag die Idee eines „europäische[n] Föderalismus innerhalb Deutschlands“1093 zugrunde, der sich mit dem auch in den Exilplänen häufig anzutreffenden doppelten Föderalismus in Deutschland und Europa deckte ${ }^{1094}$. Konnte Moltke seine Idee der fundamentalen Neuordnung des Kontinents nicht durchsetzen, so bleibt doch das eindrucksvolle Bekenntnis aller Kreisauer zum gesamteuropäischen Bundesstaat, das als Überzeugung des gesamten Kreises erstmals in den „Grundsätzen für die Neuordnung “ vom August 1943 Ausdruck fand ${ }^{1095}$. In sozialdemokratischer Diktion beschrieb Theodor Steltzer

union bis zum Ural als „Schutzbefohlene“ ohne mitgliedschaftliches Vollrecht: Moltke, Ausgangslage und Aufgaben, 9.6. 1941, abgedruckt in: LIPGENS, Föderationspläne 1968, 117-121 (118).

1089 Moltke, Ausgangslage, Ziele und Aufgaben, 24.4. 1941, abgedruckt in: RooN, Neuordnung im Widerstand 1967, S. 513.

1090 Ibid.

1091 Ibid. S. 512.

1092 Trott, handschriftliche Notizen in den USA, Winter 1939/40, abgedruckt in: LIPGENS, Föderationspläne 1968, S. 105f. (106).

1093 Trott, Denkschrift für Sir Stafford Cripps, Ende April 1942, abgedruckt in: LIPGENS, Föderationspläne 1968, S. 125-128 (127).

1094 In Verkennung des Initiativpotentials idealistischer Programmatik urteilt GRAML, Die außenpolitischen Vorstellungen 1966, S. 52 f.: „Eben weil er sie als, notwendige Einrichtungen' ansah, verstand Trott auch besser als Moltke, daß die Existenz und die Souveränität der Staaten Realitäten sind, die man höchstens benutzen, nicht aber einfach liquidieren kann."

1095 Die Kreisauer „Grundsätze für die Neuordnung“, 9. 8. 1943, abgedruckt in: LIPGENS, Föderationspläne 1968, S. 153 ff.: „Die freie und friedliche Entfaltung nationaler Kultur ist mit der Aufrechterhaltung absoluter einzelstaatlicher Souveränität nicht mehr zu vereinbaren. Der Friede erfordert die Schaffung einer die einzelnen Staaten umfassenden Ordnung. Sobald die freie Zustimmung aller beteiligten Völker gewährleistet ist, muß den Trägern dieser Ordnung das Recht zustehen, auch von jedem einzel- 
fünf Tage vor Stauffenbergs Attentat auf Hitler den europäischen Bundesstaat als Ausdruck des Genossenschaftsgedankens: „Wir müssen jetzt lernen, von einer realistischen Beurteilung der Wirklichkeit auszugehen. Ihre Konsequenz kann nur die Erkenntnis sein, daß Deutschland in Zukunft gar nicht mehr die Möglichkeit hat, machtmäßig und rohstoffmäßig als souveräner Einzelstaat weiter zu existieren und seine Stellung und Sicherung nur als genossenschaftliches Mitglied einer größeren Völkerorganisation finden kann."1096

In institutioneller Hinsicht präferierte der Kreisauer Kreis gemäß Moltkes dem Subsidiaritätsgedanken entlehnten Verdikt der kleinen Gemeinschaften einen Aufbau von unten mit möglichst umfangreichen Zuständigkeiten der nicht souveränen Selbstverwaltungseinheiten ${ }^{1097}$. In Moltkes Europaplan verfügte der europäische Bundesstaat über eine starke Exekutive in der Gestalt einer europäischen Regierung mit mindestens fünf Mitgliedern: dem Ministerpräsidenten und je einem Ressortleiter für Außenpolitik, Wehrangelegenheiten, Inneres und Wirtschaft. Unterstützung würde das europäische Kabinett durch ein gesamteuropäisches ständiges Sekretariat sowie eine gesamteuropäische Planwirtschaftsbehörde erhalten ${ }^{1098}$. Deren Kontrolle sollte einem gesamteuropäischen höchsten Gericht als Judikative und einer $z$ weigeteilten Legislative übertragen werden. Moltke befürwortete ein Zweikammersystem mit einer direkt gewählten europäischen Völkervertretung und einem Gesandtentag der europäischen Staaten ${ }^{1099}$. Dem Gesandtentag würden Vertreter der Länderregierungen angehören, denen lediglich eine beratende Funktion zukommen sollte ${ }^{1100}$.

Bis 1943 ging Moltke von einer europäischen Eigendynamik aus, die die Kriegsgegner in allen europäischen Staaten an die Spitze bringen würde. Diese würden sich dann untereinander auf einen "wahrhaft europäischen Friedensschluß“ einigen ${ }^{1101}$. Zu dieser Zeit erwartete Moltke, der das ordnungspolitische Machtpotential Großbritanniens lange überschätzte, eine „Fusion" des britischen Empire mit den USA, wodurch die „unbestrittene und unbestreitbare Vorherrschaft" wieder aufgerichtet würde, die die Voraussetzung für einen stabilen Frieden sei1102. Darin spiegelt sich die ausge-

nen Gehorsam, Ehrfurcht, notfalls auch den Einsatz von Leben und Eigentum für die höchste politische Autorität der Völkergemeinschaft zu fordern. “

1096 Steltzer, Denkschrift an englische Freunde, 15. 7. 1944, abgedruckt in: LIPGENS, Föderationspläne 1968, S. 168-170 (169f.).

1097 Vgl. die o. g. Zitate zur Einschränkung der Souveränität sowie RoON, Neuordnung im Widerstand 1967, S. 455.

1098 Moltke, Ausgangslage, Ziele und Aufgaben, 24.4. 1941, abgedruckt in: RoON, Neuordnung im Widerstand 1967, S. 517.

1099 Ibid.

1100 Ibid. S. 513.

1101 Zit. n. MommsEN, Künftige Neuordnung 1994, S. 253.

1102 Brief Moltkes an seine Frau, 6. 11. 1940, zit. n. Roon, Neuordnung im Widerstand 1967, S. 464. In seiner Denkschrift sprach Moltke von einer „angelsächsische[n] 
prägte Westorientierung, die der Kreisauer Kreis als nahezu einzige Widerstandsgruppe des innerdeutschen Widerstandes verfocht $t^{1103}$, während der übrige bürgerliche Widerstand der Idee eines „dritten Weges“ Deutschlands zwischen Ost und West nachhing ${ }^{1104}$. Bezeichnenderweise setzten sich die Kreisauer auch intensiver als andere innerdeutsche Widerstandsgruppen mit dem Freiheitsbegriff auseinander ${ }^{1105}$.

Als sich aber im Verlaufe des Jahres 1943 der Sieg der Alliierten unter dem beherrschenden Einfluß der außereuropäischen Großmächte abzeichnete, erkannte Moltke das west-östliche Spannungsfeld und plädierte noch im Frühjahr 1943 für einen deutschen Entspannungsbeitrag „zur Verhinderung einer organisatorischen Vergewaltigung des europäischen Lebens"1106. Damit entsprach er Trotts Vorstellung von einer Brücke zwischen dem „Realprinzip des Ostens" und dem „Personalprinzip des Westens“1107. Nachdem Großbritannien sichtbar von den beiden außereuropäischen Partnern faktisch entmachtet worden war und die wesentlichen Entscheidungen nunmehr allein zwischen Stalin und Roosevelt gefällt wurden, blieb dem Widerstandskämpfer nur die Hoffnung, daß die Sieger zumindest von dem „einsetzenden Neuordnungsimpuls" erfaßt würden ${ }^{1108}$.

Auch mit der Frage einer Weltorganisation beschäftigte sich der Kreisauer Kreis verschiedentlich. Grundsätzlich war man der Auffassung, daß die europäische Neuordnung einer neuen Weltordnung zeitlich voranzugehen habe ${ }^{1109}$. Insbesondere in wirtschaftlicher Hinsicht betrachtete man diese zeitliche Reihenfolge als unabdingbar: „Die wirtschaftliche Ordnung Europas ist die Voraussetzung für eine wirtschaftliche Friedensordnung der Welt, in die sich Europa durch Beteiligung am Welthandel eingliedert. "1110 Unmittelbar vor der Teheraner Konferenz warnte von Trott zu Solz davor, in den sich abzeichnenden Plänen der Alliierten für eine Weltorganisation ein omnipotentes Allheilmittel zu sehen ${ }^{1111}$. Trott kritisierte vor allem die einseitige

Union" mit wirtschaftlichem Schwerpunkt in den USA: Moltke, Ausgangslage, Ziele und Aufgaben, 24. 4. 1941, abgedruckt in: RoON, Neuordnung im Widerstand 1967, S. 512.

1103 Vgl. MEHRINGER, Widerstand und Emigration 1997, S. 193.

1104 Vgl. zu dieser typisch konservativen Denkfigur HAMmERSEN, Politisches Denken im Widerstand 1993, S. $91 \mathrm{ff}$.

1105 Ibid. S. 110-114.

1106 Denkschrift „Außen- und Innenpolitik“, zit. n. MOMMSEN, Künftige Neuordnung 1994, S. 253.

1107 Zit. n. MOMMSEN, Künftige Neuordnung 1994, S. 258.

1108 Ibid. S. 253.

1109 RoON, Neuordnung im Widerstand 1967, S. 467.

1110 Moltke, Fragestellung zur Wirtschaftspolitik in ihrer Beziehung zur Außenpolitik, abgedruckt in: ROON, Neuordnung im Widerstand 1967, S. 552 f. (552). Ein weiterer Abdruck ist enthalten in: LIPGENS, Föderationspläne 1968, S. 150-152.

1111 Trott, Bemerkungen zum Friedensprogramm der amerikanischen Kirchen, November 1943, abgedruckt in: RoON, Neuordnung im Widerstand 1967, S. 578-582 
Fokussierung auf Weltpolizei und Rüstungsbeschränkung, die er als die „negativen Mittel der Friedenssicherung" bezeichnete. Damit machte der Diplomat auf die strikt repressive Ausrichtung des Konzeptes der „Vier Weltpolizisten" aufmerksam und mahnte eine Ergänzung um die Instrumente präventiver Konfliktverhütung an. Hierzu zählte Trott in erster Linie „die ständige Betätigung praktischer und konstruktiver Zusammenarbeit zwischen den Nationen"1112, die er zu Recht in der alliierten Konzeption vermißte.

Im Gegensatz zum Kreisauer Kreis vertrat der Goerdeler-Kreis unverhohlen die Positionen einer großdeutschen Machtstaatspolitik. Innenpolitisch favorisierte man zumindest für eine Übergangszeit ein autoritäres Regime ${ }^{1113}$. $\mathrm{Da}$ die „Honoratioren“ das Deutsche Reich als europäische Ordnungsmacht par excellence ansahen, konnten und wollten sie sich Europa nur unter deutscher Hegemonie vorstellen. Mit dem deutschen Sieg über Frankreich wurde die nationalkonservative Tendenz noch einmal gestärkt ${ }^{1114}$. So formulierte Goerdeler selbst in einer an den britischen Außenminister gerichteten Denkschrift über die "Grundsätze für Friedenswirtschaft" vom Oktober 1940: "Genau wie einst Preußen im preußischen Zollverein und im Norddeutschen Bunde muß heute Deutschland im neuen Europa den sichtbaren Vorteil der neuen Ordnung als Anziehungskraft auf die freie Entschließung der Völker wirken lassen, um sie für diese Ordnung und für eine deutsche Führung [sic!] zu gewinnen." 1115

Anders fiel Goerdelers Beurteilung einer wirtschaftlichen Hegemonie Deutschlands aus. Hier erkannte er das immense Konfliktpotential einer von Berlin aus gelenkten europäischen Wirtschaft, „weil sie auf die in der Seele der Völker verankerten nationalen Empfindungen stoßen müßte" ${ }^{1116}$. Es bleibt offen, welche Gründe Goerdeler daran gehindert haben, diese realistische Einschätzung der Vorbehalte gegen ein deutsch dominiertes Europa von der ökonomischen auf die politische Ebene zu übertragen ${ }^{1117}$. Jedenfalls entwickelte Goerdeler bis 1943 das Konzept eines „nur" politisch von Deutschland dominierten Wirtschaftsraumes Europa, der nach und nach zu einem

(581). Das Dokument ist auch abgedruckt in: LIPGENS, Föderationspläne 1968, S. 162165.

1112 Ibid.

1113 Fragwürdig erscheint die These KLEMPERERs, „Verbindung zu der großen Welt“ 1990, S. 10, der deutsche Widerstand habe die Forderung nach einem autoritären Regime nach Hitler lediglich aus Rücksicht auf die seiner Ansicht nach unverzichtbare Mitwirkung der zögernden Generäle erhoben. Jedenfalls bei Goerdeler dürfte dahinter ein tatsächlicher Glaube an die Organisationskraft des Autoritären gestanden haben. 1114 FEST, Staatsstreich 1994, S. 166, spricht von „tief im Bewußtsein verankerten Vormachtreflexe[n]".

1115 Goerdeler, Grundsätze für Friedenswirtschaft, Oktober 1940, abgedruckt in: LIPGENS, Föderationspläne 1968, S. 109 ff. (110).

1116 Ibid. S. $110 f$.

1117 LIPGENS, Föderationspläne 1968, S. 109 verweist auf Goerdelers Balkanreise 1938, die ihn bewogen haben soll, sich in ökonomischer Hinsicht für einen europäischen $\mathrm{Zu}$ sammenschluß auszusprechen. 
Militärbund erweitert werden sollte. In der Denkschrift „Das Ziel“ vertrat der ehemalige Kommunalpolitiker diese Positionen letztmalig in uneingeschränktem Umfang und verstieg sich zu der Behauptung, "daß bei rechtzeitigem Handeln, d.h. Abbruch des Krieges zugunsten eines sinnvollen politischen Systems, der europäische Staatenbund unter deutscher Führung in 10 bis 20 Jahren Tatsache sein wird"1118. Ebenso dachte der 1938 zurückgetretene Chef des Generalstabs des Heeres, Ludwig Beck, an einen europäischen Wirtschaftszusammenschluß, blieb darüber hinaus jedoch ebenso im konventionellen Nationalstaatsdenken verhaftet wie Goerdeler zu dieser Zeit ${ }^{119}$.

Goerdelers strikt nationalstaatliches Denken erfuhr durch die deutschen Niederlagen des Jahres 1942 und sicherlich auch durch die wachsende Einsicht in den logischen Bruch zwischen seinem außenpolitischen und dem außenwirtschaftlichen Konzept eine grundlegende Umorientierung, die sich schrittweise vollzog. In einer "Erklärung zur Atlantik-Charta“ vom 13. Dezember 1942 verzichtete Goerdeler erstmals auf den deutschen Führungsanspruch in Europa, beharrte jedoch weiterhin auf der uneingeschränkten Souveränität der Nationalstaaten ${ }^{1120}$. Ausgehend von den vorhandenen nationalen Organisationen sollte schrittweise die wirtschaftliche, geistige und kulturelle Annäherung der europäischen Staaten betrieben werden ${ }^{1121}$. Obwohl Goerdeler die Errichtung neuer Organisationen grundsätzlich ablehnte, schlug er die Schaffung eines ständig tagenden europäischen Wirtschaftsrates vor, dessen Aufgaben in der Vereinfachung des Grenzverkehrs, dem allmählichen Abbau der Zollschranken, der Anbahnung einheitlicher Bestimmungen für Verkehr und Telekommunikation sowie der Rechtsangleichung im Handelsrecht, Wechselrecht und Bürgerlichem Recht bestehen sollten ${ }^{1122}$. Statt der seines Erachtens zu hoch gesteckten Ziele des alten Völkerbundes sprach sich der ehemalige Oberbürgermeister von Leipzig für die Minimalversion einer intergouvernemental strukturierten Weltorganisation aus, die er sich als "ein ständig arbeitendes kleines Büro" vorstellte mit der einzigen Aufgabe, Zusammenarbeit und Vertrauen unter den Völkern zu fördern ${ }^{123}$. An die Bildung einer Völkerbundsexekutive sei erst zu denken, „wenn in Europa eine gewisse Stufe der Zusammenarbeit erreicht und für Europa eine europäische Polizeigewalt stabilisiert ist" ${ }^{1124}$.

Mitte 1943 - möglicherweise beschleunigt durch das Treffen mit dem dezi-

1118 Goerdeler, Das Ziel, abgedruckt in: LIPGENS, Föderationspläne 1968, S. $123 \mathrm{ff}$. (125).

1119 Beck, Voraussetzungen und Grundsätze, Juni 1942, abgedruckt in: LIPGENS, Föderationspläne 1968, S. $136 \mathrm{f}$.

1120 Goerdeler, Erklärung zur Atlantik-Charta, 13. 12. 1942, abgedruckt in: LIPGENS, Föderationspläne 1968, S. 143-146 (144).

1121 Ibid. S. 145.

1122 Ibid.

1123 Ibid.

1124 Ibid. S. $145 \mathrm{f}$. 
diert europaföderalistischen Kreisauer Kreis vom 8. Januar 19431125 - wandte sich Goerdeler ausdrücklich vom deutschen Hegemonialanspruch ab und propagierte nunmehr das Ziel einer europäischen Föderation gleichberechtigter Mitgliedstaaten: Europa müsse sich „zu einem ewigen Friedensbund zusammenfinden, in dem weder Deutschland noch eine andere Macht Vorherrschaft beansprucht"1126. Dabei betrachtete er den europäischen Zusammenschluß auch als „Sicherung gegen [die] russische Übermacht“1127, optimalerweise garantiert durch eine natürliche Interessengemeinschaft Deutschlands und Großbritanniens, denn: „Man wird damit rechnen müssen, daß Amerika nicht immer die Sicherung Europas gegenüber Rußland mit übernimmt. " 1128 Nun erfuhr auch die Idee des ursprünglich intergouvernementalen europäischen Wirtschaftsrates eine bedeutende Weiterentwicklung zu einem europäischen Wirtschaftsministerium. Mit dem Vorschlag, dem gemeinsamen Wirtschaftsministerium ein europäisches Außenministerium zur Seite zu geben und eine europäische Wehrmacht aufzustellen ${ }^{1129}$, befreite sich Goerdeler von den Fesseln nationalstaatlicher Blickfeldverengung. Gutgläubig ging er davon aus, daß man sich über Einzelheiten unschwer werde verständigen können ${ }^{1130}$.

Mit dem Anfang 1944 formulierten uneingeschränkten Europabekenntnis hatte Goerdelers beachtlicher Erkenntnisprozeß vom ehemals großdeutschhegemonialen Ansatz bis zur antihegemonialen Europaföderation schließlich seinen Abschluß gefunden. Goerdeler war nun überzeugt, daß nur „die entschlossene Anbahnung einer inneren und lebendigen Einheit Europas" dem Kontinent ermöglichen werde, nach innen Frieden zu schaffen und sich nach außen als Machtfaktor neben den "großen neuen Weltgebilde[n]" Amerika und Rußland zu behaupten. Mit Nachdruck verwies er auf die Notwendigkeit, ,endgültig die Grenzen in Europa niederzureißen und ein ebenbürtiges Gebilde zu schaffen"1131. Ähnlich dem Kreisauer Konzept der kleinen Gemeinschaften forderte Goerdeler, den europäischen Aufbau nach dem Subsidiaritätsprinzip zu organisieren ${ }^{1132}$. An der Spitze sollte ein europäischer Verwaltungsausschuß mit Spezialkommissionen bestehen, dessen Aufgabenumschreibung an das heutige Diktum von der Europäischen Kommission als dem Motor der Integration erinnert: Der sachverständige Verwaltungsausschuß sollte „durch die Jahre hindurch, wenn Streit, Kleinmut und Bedenken die große Idee dieser Tat mindern wollen, das heilige Feuer wachhalten, gu-

1125 So jedenfalls FEsT, Staatsstreich 1994, S. 168.

1126 Goerdeler, Friedensplan, [Spätsommer/Herbst] 1943, abgedruckt in: LIPGENS, Föderationspläne 1968, S. 156.

1127 Ibid.

1128 Ibid.

1129 Ibid. S. 157.

1130 Ibid.

1131 Goerdeler, Praktische Maßnahmen zur Umgestaltung Europas, [Anfang 1944], auszugsweise abgedruckt in: LiPGENS, Föderationspläne 1968, S. $165 \mathrm{ff}$. (166).

1132 Ibid. 
ten Willen stärken, Kleinmütige ermuntern, Böswillige durch Bloßstellung strafen." 1133

Zusammenfassend kann festgehalten werden, daß schon ein kursorischer Überblick über die Europavorstellungen des innerdeutschen Widerstandes wichtige Anhaltspunkte für einen Vergleich mit dem Europadiskurs des deutschen Exils bietet. Sicherlich ist weder das politische noch das soziale Umfeld des Exils mit den Umständen des innerdeutschen Widerstandes vergleichbar. Die Emigranten konnten sich - abgesehen von den nicht zu unterschätzenden Erschwernissen und Enttäuschungen eines Lebens im Exil - relativ frei bewegen, sich außerhalb der Zensur ungehindert austauschen und erlebten in der Regel die tägliche Praxis einer funktionierenden Demokratie mit den Grundrechten der Meinungs- und Informationsfreiheit sowie der Versammlungsfreiheit aus eigener Anschauung. Die innerdeutschen Widerstandskämpfer dagegen waren der permanenten Gefahr der Beobachtung, der Denunziation und Entdeckung ausgesetzt. Selbst das Gespräch unter Freunden bedurfte unter diesen Umständen höchster Wachsamkeit. Auch waren die Kontakte zu demokratischen Lebensformen eher sporadischer Art und beschränkten sich zumeist auf wenige Auslandsreisen. Zugleich sollte jedoch nicht vergessen werden, daß der überwiegende Teil der bürgerlichen Widerstandsgruppen in Deutschland die nationalsozialistische Innen- und Außenpolitik oft sehr lange und aktiv als Angehörige des Auswärtigen Dienstes, des Militärs oder einzelner Reichsministerien mitgetragen hat, sofern der einzelne nicht schon 1938/39 zum konspirativen Widerstand übergegangen war. Schließlich wird ein Vergleich zwischen den Europavorstellungen des inneren und des äußeren Widerstandes gegen das Hitler-Regime dadurch relativiert, daß der Europadiskurs des Exils ungleich mehr Teilnehmer unterschiedlichster politischer Provenienz kannte als die vergleichsweise spärlichen Äußerungen des inneren Widerstandes, die sich in der Sache auf die Kreise um Moltke und Goerdeler beschränken. Dies umso mehr als das Exil ja gerade kein einheitliches Europakonzept erarbeitet hatte, sondern sich durch eine Vielfalt an Ideen und Entwürfen auszeichnete. So finden sich die Charakteristika der nationalkonservativen Europaentwürfe aus dem Goerdeler-Kreis wie der großdeutsch-revisionistische Ansatz, das Denken in traditionellen Autoritätskategorien und der deutsche Führungsanspruch nicht nur bei Otto Strasser oder Hermann Rauschning wieder, sondern auch bei gemäßigten Emigranten. Die Schlüsselposition Deutschlands im künftigen Europa, die häufig nicht weit vom deutschen Hegemonialdenken entfernt war, wurde selbst in sozialistischen Kreisen lange Zeit hochgehalten, und der Erhalt des nationalstaatlichen Ordnungsprinzips in Europa unter Verzicht auf jedes supranationale Element hatte in nahezu allen politischen Exilgruppen Anhänger, die bis 1943 - von Ausnahmen wie dem ISK abgesehen sogar die Mehrheit stellten.

1133 Ibid. S. 167. 
Gemeinsam war dem mit der Zukunft Europas beschäftigten deutschen Widerstand im Inland und Ausland nicht nur der Wunsch, der Welt die Existenz des "Anderen Deutschland" zu beweisen. Vielmehr waren Exil und innerdeutscher Widerstand nahezu unabhängig voneinander einem Prozeß der innereuropäischen Rückbesinnung ausgesetzt, an dessen Ende häufig die Überzeugung stand, daß nur eine europäische Föderation den Frieden in Europa retten könne ${ }^{1134}$. Diese parallele Entwicklung war auch bedingt durch die gleichartigen Motivbündel, die zur Auseinandersetzung mit der europäischen Zukunft antrieben. Friedenssicherung, wirtschaftliche Prosperität, antibolschewistische Schutzmechanismen und die Bewahrung des abendländischen Kulturraumes spielten in beiden Ausprägungen des deutschen Widerstandes eine zentrale Rolle. Mit der zunehmenden Entscheidungsgewalt außereuropäischer Staaten über die Zukunft Europas trat der Aspekt der europäischen Unabhängigkeit im sich anbahnenden Ost-West-Konflikt in den Vordergrund der Erörterungen. Zugleich war man im In- und Ausland gezwungen, sich mit den alliierten Plänen einer Weltorganisation und deren Verhältnis zu einer in der Regel gleichzeitig angestrebten europäischen Föderation auseinanderzusetzen. Zum Kriegsende hin kam schließlich verstärkt die Sorge um den Erhalt Deutschlands als einem einheitlichen Staatsgebilde hinzu.

Legt man die betont föderalistischen Europapläne des Kreisauer Kreises zugrunde, ergeben sich weitergehende Parallelen zu vielen Entwürfen des Exils. So traf die zentrale Forderung nach einem europäischen Bundesstaat jedenfalls nach der Teheraner Konferenz in nahezu allen Exilgruppen auf lebhafte Zustimmung, auch wenn in vielen Kreisen des Exils ein langer Denkprozeß vorangegangen war. Auch der dezidierte Antinationalismus, der eng mit der Frage einer europäischen Souveränität verknüpft war, findet sich als grundlegendes Motiv und wegweisende Erkenntnis in den Debatten des Exils wieder. Schließlich stimmten die Kreisauer in ihrem Ruf nach Schaffung eines europäischen Kollektivbewußtseins auf der Grundlage der gemeinsamen Werttraditionen mit einer Vielzahl von Europakonzeptionen des Exils überein. Nicht nur die sozialistischen Gruppen erinnerten an die gemeinsamen europäischen Wurzeln und die gemeinsame Interessenlage der Zukunft. Auch bürgerliche Emigranten wie Wilhelm Röpke, der ein "geistig-morali-

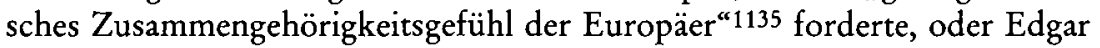
Stern-Rubarth, der an den europäischen Patriotismus appellierte ${ }^{1136}$, mahnten eine Änderung der ordnungspolitischen Identifikation an.

Stern-Rubarths Appell an einen neuen, europäischen Patriotismus, der sich von der Nation als Identifikationsobjekt lösen müsse, ist zugleich ein lehrreiches Beispiel für die oft frappierenden Parallelen zwischen dem Euro-

$1134 \mathrm{Zu}$ einem ähnlichen Ergebnis kommt KLEMPERER, „Verbindung zu der großen Welt“ 1990, S. 19.

1135 Röpke, in: VoIGT, Friedenssicherung 1988, S. 174.

1136 STERN-RUBARTH, Exit Prussia 1940, S. 164. 
padiskurs des deutschen Exils und den Europadiskursen des übrigen europäischen Widerstandes in den vom Nationalsozialismus unterdrückten Ländern. Stern-Rubarth hatte in diesem Zusammenhang daran erinnert, daß sich der geographische Raum des Patriotismus ständig vergrößert habe ${ }^{1137}$. Nahezu gleichlautend ließ sich die französische Résistance vernehmen: „Die Geschichte lehrt uns eine ständige Ausweitung der Grenzen. ${ }^{~}{ }^{1138}$ Solche parallelen Erkenntnisprozesse waren weder zufällig noch hatten sie Ausnahmecharakter. Henri Frenay sprach wohl zu Recht von der „Internationale des Widerstandes“ - und meinte damit das verbindende Element des „Europas des Schmerzes, das den Tag in Angst beginnt, in jenem Untergrund-Europa des Maquis und der falschen Papiere, in jenem blutenden Europa, das geschlagen wird und Schlag zurückgibt" ${ }^{1139}$.

Der engagierte Europaföderalist Willi Eichler bemerkte in einem Rundschreiben an die Funktionäre des ISK im August 1943: „Gerade in den letzten Wochen haben beinahe sämtliche illegale Bewegungen Europas ihre grundsätzliche Stellungnahme zu einer Reihe wichtiger politischer Fragen veröffentlicht, darunter selbstverständlich jedes Mal auch eine Stellungnahme zu der Frage einer Regelung europäischer Probleme und zu der Frage, was man nach dem Kriege mit Deutschland tun solle. [...] Die sind alle der Meinung, daß Europa irgendwie vereinheitlicht werden muß, daß es darin Gleichberechtigung und möglichst große Freiheit geben muß und daß man sich selbstverständlich vor weiteren deutschen Angriffen unter allen Umständen schützen muß, daß aber die freiheitlichen Kräfte Deutschlands gestützt werden sollten, um aus Deutschland einen freiheitlichen Staat zu machen, der im Rahmen einer europäischen Völkergemeinschaft einen ernsthaften Platz einzunehmen hat. "1140 Damit hatte Eichler die wesentlichen Übereinstimmungen des europäischen Widerstandes umschrieben, auch wenn sein Blick auf die sozialistischen Gesinnungsgenossen Europas verengt war. Die Überzeugungen weitblickender Widerstandskämpfer wie Altiero Spinelli in Italien, Léon Blum und Henri Frenay für die französische Résistance, Ronald Mackay in Großbritannien und dem Generalsekretär des Weltkirchenrates in Genf, Willem Visser't Hooft, trafen sich in vielen Details, vor allem aber in der gemeinsamen Einsicht, daß eine europäische Föderation das geeignetste Mittel sei, den Frieden in Europa langfristig zu sichern ${ }^{1141}$. Daß

\section{Ibid.}

1138 LIPGENS, Europäische Verfassung 1986, Dok. Nr. 20, S. 91.

1139 FrenaY, in: Combat, Nr. 53 (Dezember 1943), wiedergegeben in: LIPGENS, Föderationspläne 1968, S. $229 \mathrm{f}$., Anm. 3.

1140 [Eichler], Rundschreiben des Bundesvorstandes an die Funktionäre, August 1943, abgedruckt in: EIBER, Union 1998, Dok. Nr. 312, S. 770-778 (771).

1141 Die folgenden Ausführungen können exemplarisch an sechs Dokumenten des europäischen Widerstandes nachgewiesen werden: Altiero Spinelli, Das Manifest von Ventotene, Juli 1941, abgedruckt in: LIPGENS, Föderationspläne 1968, S. 36-44; Léon Blum, À l'échelle humaine, Juli 1941, abgedruckt in: LIPGENS, ibid. S. 185-190; [Comité Général d'Études], Frankreich im Europa von morgen, Januar 1944, abgedruckt in: Lip- 
ein vereinigtes Europa zugleich die innere Freiheit der Demokratie und die äußere Freiheit des Kontinents von außereuropäischen Einflüssen am ehesten garantieren könne, davon waren sie alle überzeugt. Und selbst in der Frage, wie Europa vor künftigen Aggressionen Deutschlands zu schützen sei, erfuhren die deutschen Emigranten internationale Zustimmung in ihrer mehrheitlich vertretenen Position, daß Deutschland im europäischen Interesse möglichst bald in einen Europabund integriert werden müsse. Die europäischen Widerstandsgruppen wurden getragen durch den gemeinsamen Willen, aus den Schwächen des Völkerbundes zu lernen. Geeint durch den „ewige[n] Wert des kritischen Geistes"1142, teilten sie die Erkenntnis, daß das Versagen des nationalstaatlichen Ordnungsprinzips durch den überzogenen Nationalismus endgültig sichtbar geworden war und durch eine übernationale Identität abgelöst werden mußte. Schließlich waren sich die europäischen Widerstandskämpfer einig, daß der seit 1918 als Notwendigkeit erkannte wirtschaftliche Zusammenschluß nun endlich verwirklicht werden müsse, sei es als Auftakt zu einer politischen Union oder lediglich als Abwehrmechanismus gegen die weltwirtschaftliche Konkurrenz, namentlich der USA.

\section{Ergebnisse}

„Nicht nur gegen Hitler, sondern auch für Europa“ - mit der Verknüpfung der beiden Hauptaufgaben der politischen Emigration war der Minimalkonsens im Europadiskurs der deutschen Exilgruppen gefunden: die Überwindung des nationalsozialistischen Regimes und seine Auflösung in einer Neuordnung Europas. Nachdem die deutschen Emigranten in den Jahren von 1933 bis 1937 das Widerstandspotential des Europagedankens entdeckt hatten, bemühten sie sich nach dem Münchner Abkommen um dessen gezielte Einbindung in den Kampf gegen den Nationalsozialismus.

1938 erreichte der Prozeß der innereuropäischen Rückbesinnung eine neue Phase der Konkretisierung und politischen Inanspruchnahme der bis dahin vagen Europaideen des deutschen Exils. Die Motive für einen Zusammenschluß der europäischen Staaten waren bald gefunden: langfristige Sicherung des Friedens in Europa und damit in der Welt, wirtschaftliche Prosperität zur Vermeidung künftiger sozialer Konflikte sowie die von allen Exilgruppen bemühten Schlagworte der Freiheit und der Demokratie als Garanten für die gemeinsame Gestaltung einer friedlichen europäischen Zukunft.

GENS, ibid. S. 231 f.; Ronald W. G. Mackay, Entwurf der Verfassung der Vereinigten Staaten von Europa, [Juli] 1940, abgedruckt in: LIPGENS, ibid. S. 423-436 und die beiden Deklarationen der Genfer Konferenz von Widerstandskämpfern um Willem Visser't Hooft vom 20. Mai 1944, abgedruckt in: LIPGENS, ibid. S. 392-398.

1142 Spinelli, Das Manifest von Ventotene, Juli 1941, abgedruckt in: LIPGENS, Föderationspläne 1968, S. 36-44 (38). 